difficult to avoid tartrazine or sodium benzoate. One large food manufacturer does not use tartrazine in his products; perhaps others can be persuaded to find satisfactory alternatives. Nevertheless, despite the practical difficulties a few months on a diet low in a substance which has been shown to aggravate the urticaria in a particular individual is well worth the effort required. Not only is there usually immediate symptomatic relief, but after a few months of strict adherence to the diet some relaxation may be allowed, and the offending substance may then be found to be tolerated.

1 Warin, R P, and Champion, R H, Urticaria. Philadelphia, Saunders, 1974.

- Champion, R H, et al, British fournal of Dermatology, 1969, 81, 588.

Tas, J, Dermatologica, 1967, 135, 90.

Thompson, J S, Annals of Internal Medicine, 1968, 69, 361

Calnan, C D, Lancet, 1957, 1, 996.

${ }^{6}$ Moore-Robinson, M, and Warin, R P, British Medical fournal, 1967, 4, 262

' Michaëlsson, G, and Juhlin, L, British fournal of Dermatology, 1973, 88, 525

'Doeglas, H M G, British fournal of Dermatology, 1975, 93, 135.

${ }^{9}$ Warin, R P, and Smith, R J, British fournal of Dermatology, 1976, 94, 401.

\section{New look at monoamine oxidase inhibitors}

Despite nearly 20 years' clinical use, the place of the monoamine oxidase inhibitor drugs (MAOIs) in psychiatry remains uncertain. They are inconsistently prescribed-a panacea to some practitioners, poison to others. Recently interest in them has been revived. ${ }^{1}$

Though they are used much less than the tricyclic antidepressives, the MAOIs have been claimed to have special value in some psychiatric syndromes. These include reactive, neurotic, or atypical depressive conditions; depressive-anxiety states; phobic anxiety; and phobic anxiety-depersonalization syndromes. The few controlled studies made have indicated that patients in hospital with typical depressive illnesses of moderate or severe intensity fare badly with MAOI treatment. ${ }^{23}$ Depressed outpatients do better: in one trial ${ }^{4}$ phenelzine was effective in outpatients over the age of 35 with atypical features such as anxiety, fatigue, phobias, and somatic complaints.

In reported trials, depressed patients who improved with MAOI therapy had unequal changes in their symptoms. Subjective disturbances (both depression and anxiety) did not improve with phenelzine as compared with placebo; on the other hand, retardation, agitation, and somatic symptoms of anxiety were usefully controlled. ${ }^{+}$Patients with longstanding phobic anxiety also respond to MAOIs. In one study ${ }^{5}$ those receiving an average daily dose of $45 \mathrm{mg}$ of phenelzine improved significantly more than those treated with placebo, both on overall assessment and in their work record. General anxiety and panic also decreased. Patients with phobia and depression tended to improve less than those not depressed. Tyrer et al suggested that phenelzine acts in phobic patients as an anxiolytic rather than an antidepressive. Similarly, iproniazid has anxiolytic effects in severely agoraphobic patients. ${ }^{6}$ Relapse is likely to occur if the MAOI is withdrawn.

The time before clinical response occurs ranges from a few days to several weeks, tranylcypromine usually acting more rapidly than phenelzine or isocarboxazid. There is a delay before REM sleep is suppressed by MAOIs, and the time of this suppression apparently coincides with clinical improve- ment. ${ }^{7}$ Among the MAOIs, phenelzine and tranylcypromine are generally thought most effective, the former having the lower reported incidence of hypertensive crises. Though this group of drugs has a reputation as being dangerously toxic, from his extensive experience Tyrer" has concluded that "they are generally safe provided that proper caution is exercised with dietary and drug intake."

Despite their clear biochemical properties there have been surpi singly few attempts to relate the actions of these drugs to the clinical response produced. Amine concentrations in brain tissue taken post mortem from patients maintained on MAOIs in their terminal illnesses have generally been found to be raised. Nevertheless, about a quarter of these specimens showed no evidence of MAO inhibition at all. ${ }^{8}$ Therapeutically, estimating the MAO activity in the blood platelets or urinary tryptamine excretion may provide information on peripheral MAO inhibition. Though the relation between peripheral and central MAO inhibition is not clear, the success of the MAOIs in one trial ${ }^{4}$ may have been due to careful monitoring of platelet $\mathrm{MAO}$ and the adjustment of drug dosage to maintain an inhibition of at least $80 \%$. Phenelzine is metabolised by acetylation, and people in whom this process is slow ("slow acetylators") are quicker to attain high degrees of MAO inhibition and show a more prompt clinical response than fast acetylators. ${ }^{9}$ Hence some of the variations in clinical response and contradictions in the results of clinical trials may be due to pharmacokinetic differences among individuals.

Finally, there is the vexed question of whether MAOIs should be combined with tricyclic antidepressive agents. Some have hailed such combinations as most effective; others have stated that they are hazardous; uncontrolled studies of the combination have failed to show convincing evidence of their value. Nevertheless, at present two controlled trials of combined treatment are under way in London teaching hospitals which should help to settle the matter.

The resurgence of interest in the MAOIs is timely. Other types of treatments for depression and phobias are far from satisfactory, especially in those patients who seem to respond to MAOIs. More comparative evaluations with proper biochemical control are still needed.

\footnotetext{
Tyrer, P, British Fournal of Psychiatry, 1976, 128, 354

2 Medical Research Council Clinical Psychiatry Committee, British Medical Fournal, 1965, 1, 881.

${ }^{3}$ Raskin, A, et al, Archives of General Psychiatry, 1974, 30, 66.

${ }^{4}$ Robinson, D S, et al, Archives of General Psychiatry, 1973, 29, 407.

Tyrer, P, Candy, J, and Kelly, D, Psychopharmacologia, 1973, 32, 237.

Lipsedge, M S, et al, Psychopharmacologia, 1973, 32, 67.

' Dunleavy, D L F, and Oswald, I, Archives of General Psychiatry, 1973, 28, 353.

Bevan Jones, A B, et al, British Medical fournal, 1972, 1, 17.

${ }^{9}$ Johnstone, E C, Psychopharmacologia, 1976, 46, 289.
}

\section{Curry kidney}

The frequency of renal calculi, and indeed of renal disease, varies in different countries. Climate, the composition of the water, personal habits of eating and drinking, and the genetic background of the population have been invoked to explain these differences. In Fiji the Indians, who came originally from all parts of the Indian subcontinent and are therefore of varied ethnic origin, make up some half of the population; yet they are virtually the only sufferers from stones. ${ }^{12}$ All 16 operations for kidney stones at Lautoka Hospital in 1969, for instance, 
were performed on Indians ${ }^{1}$; and 74 of the 79 patients who had an intravenous pyelogram done for suspected stone were Indian, as were 26 of the 28 with unequivocal evidence of stone. Metabolic studies have recently been carried out in a group of 31 of these Indian stone formers. ${ }^{2}$ The handling of ca.cium, uric acid, and amino-acids seems to be normal, and there is nothing to suggest any inborn or acquired tubular dysfunction.

The explanation may be a matter of diet. These Fijian Indian patients may be similar to those reported by Murphy, ${ }^{34}$ in whom renal disease was attributed to the excessive use of Worcestershire sauce. His five patients had evidence of both glomerular and tubular damage, with an occasional finding of abnormal aminoaciduria and an impaired response to an ammonium chloride load. Some of his patients had stones, but one was referred to hospital for hypertension and another for renal insufficiency. Nevertheless, Worcestershire sauce contains ingredients widely eaten by Indians-garlic, black pepper, ginger, and cinnamon-and it has been suggested that the volatile oils from these may be nephrotoxic. The exact quantities of Worcestershire sauce consumed by Murphy's patients were not given in his papers, but the Fijian Indians have been estimated to eat the equivalent of a bottle of Worcestershire sauce every day in the form of curries, spices, and pickles. Though the "curry kidney" has yet to be proved as a pathological entity, the hypothesis might well be tested in other communities with a high incidence of renal stones. How common is stone, for instance, among the Asian population in Britain?

${ }^{1}$ Holmes, G, Medical fournal of Australia, 1971, 2, 755.

2 Johnson, J R, and Holmes, G, Medical fournal of Australia, 1976, 1, 521.

${ }^{3}$ Murphy, K J, Lancet, 1967, 2, 401.

${ }^{4}$ Murphy, K J, Medical fournal of Australia, 1971, 1, 1119.

\section{Abortion and maternal deaths}

In England and Wales abortion remains the most common cause of death associated with pregnancy. ${ }^{1}$ In the past these deaths mostly followed illegal or spontaneous abortion, but since the introduction of the Abortion Act 1967 deaths associated with spontaneous abortion have almost disappeared and those after illegal abortion have fallen steadily. To some extent this welcome improvement has been offset by a persistent rise in deaths associated with legal termination of pregnancy, and at present there are as many fatalities after legal as illegal abortion. ${ }^{1}$

It is disappointing that women should continue to hazard their lives by resorting to criminal or self-induced abortion, and the circumstances leading to these deaths merit close study. Of the 38 women who died after illegal abortion between 1970 and 1972, eighteen were immigrants from the New Commonwealth. ${ }^{1}$ These women may not have known that they could have obtained legal abortion in Britain or how they should have gone about it. Others might have brought with them attitudes and practices that are common in their countries of origin. Whatever the reason these women are specially at risk. Another group seem determined at any cost to keep their pregnancies secret and purposely avoid contact with family doctor or gynaecologist, ill-advisedly resorting to self-induced or criminal abortion.

Deaths associated with legal abortion have risen steadily with the rising number of abortions performed since the
Abortion Act. Tietze and Murstein ${ }^{23}$ estimated that the mortality from legal abortions in England and Wales (including deaths associated with legal abortion but not attributed to it) during $1970-3$ was $12 \cdot 3$ per 100000 abortions. The comparable figure for the United States over the same period was 6.3. To a large extent this difference may be explained by the higher average age of women obtaining abortions in Britain, the longer average duration of pregnancy at which the abortions are performed here, and the higher proportion of abortions combined with surgical sterilisation. To what extent other factors such as the choice of procedure (curettage, suction, hysterotomy without sterilisation, utus paste, or intrauterine saline) are relevant cannot be determined from available data.

In the short term little can be done to influence the age at which women seek abortions, but much could be done to ensure that most abortions are performed in the first trimester. The primary need is for more accurate infor mation about the reasons for the present delay in reaching a decision about whether to terminate the pregnancy. To what extent is the patient, the family doctor, or the gynaecologist (indeed the whole system of referral and consultation) responsible? These and other relevant questions can be answered only by well-planned prospective studies in large, representative communities. Once we have a better understanding of the sequence of events we should be able to make acceptable recommendations that would allow sufficient time for counselling without delaying a final decision beyond the twelfth week of pregnancy. Everyone concerned, including patients, should be well warned of the dangers of procrastination.

In England and Wales the proportion of legal abortions with concurrent sterilisation fell from $23 \%$ in 1968 to $10.8 \%$ n 1973. ${ }^{2}$ The comparable figure for the United States ascertained by the Joint Program for the Study of Abortion ${ }^{2}$ in 1970-1 was $3 \cdot 7 \%$. The mortality in England and Wales was nine times higher for abortions with concurrent sterilisation than for those without. ${ }^{2}$ In some measure this high death rate is related to the fact that in almost two-thirds of all abortions with sterilisation either hysterotomy or hysterectomy was required. Again, women who are aborted and sterilised at the same time are older and their pregnancies further advanced, and these factors themselves contribute to a higher mortality. Where at all possible these surgical procedures should be avoided, for they add significantly to the risks of abortion.

These are general and important features of mortality associated with abortion. The recently published Confidential Enquiries into Maternal Deaths (1970-1972) identified avoidable factors in half the deaths from legal abortion during this period, and this form of detailed study of individual deaths is most helpful. Utus paste and laminaria tents are specially mentioned as the two methods of termination carrying the highest risk, and it is deplorable that the number of deaths attributed to the introduction of a paste into the uterus during legal abortion has risen from two during 1964-6 to seven during the three years covered by the last report.

Induced abortion will never be without some risk. Nevertheless, this may be kept to a minimum if the procedure is carried out in the first trimester under suitable conditions by an experienced operator using well-established techniques.

\footnotetext{
Department of Health and Social Security, Report on Confidential Enquiries into Maternal Deaths in England and Wales 1970-1972. London, HMSO, 1975

Induced Abortion: 1975 Factbook. New York, Population Council, 1975.

Tietze, C, 1976, personal communications.

- Tietze, C, and Lewit, S, Studies in Family Planning, 1972, 3, 97.
} 